\title{
Anode modification of polyfluorene-based polymer light-emitting devices
}

\author{
M. K. Fung, S. L. Lai, S. W. Tong, M. Y. Chan, C. S. Lee, ${ }^{\text {a) }}$ and S. T. Lee \\ Center of Super-Diamond and Advanced Films (COSDAF) and Department of Physics and Materials \\ Science, City University of Hong Kong, Hong Kong SAR, China \\ W. W. Wu, M. Inbasekaran, and J. J. O’Brien \\ Advanced Electronic Materials, The Dow Chemical Company, Midland, Michigan 48674
}

(Received 13 February 2002; accepted for publication 17 June 2002)

\begin{abstract}
A glycerol-modified poly(3,4-ethylene dioxythiophene) (PEDOT): poly(styrene sulfonate) (PSS) layer was used as an anode buffer layer in polymer light-emitting devices using poly(9,9-dioctylfluorene) (F8) as the emitter. Devices with a configuration of indium tin oxide/ PEDOT:PSS (with or without glycerol)/F8/CsF/Al were fabricated. It was found that the glycerol-modified device showed a much larger current density than the unmodified device. At an operating voltage of $6 \mathrm{~V}$, the glycerol-modified device showed a luminance of $1300 \mathrm{Cd} / \mathrm{m}^{2}$ and a current efficiency of $1.7 \mathrm{Cd} / \mathrm{A}$ compared to the corresponding values of $500 \mathrm{Cd} / \mathrm{m}^{2}$ and $1.3 \mathrm{Cd} / \mathrm{A}$ in the unmodified device. Analysis by ultraviolet spectroscopy suggests that the two devices have the same energy level structure and the performance improvement should not be due to change in the PEDOT/polymer interface. It was further found that incorporating a suitable amount of glycerol into the PEDOT:PSS layer can increase its conductivity by six times. This leads to a better balance in the hole and electron currents and thus improved device efficiency. (C) 2002 American Institute of Physics. [DOI: 10.1063/1.1499519]
\end{abstract}

The successes of $\mathrm{LiF} / \mathrm{Al}^{1-3}$ and $\mathrm{LiF} / \mathrm{Ca} / \mathrm{Al}^{1}$ cathodes in polymer light-emitting diodes (PLEDs) have prompted numerous efforts to improve hole injection at the anode side by inserting an interlayer between indium tin oxide (ITO) and the hole-transporting layer. The interlayer includes $\mathrm{LiF}^{4}$ copper phthalocyanine $(\mathrm{CuPc}){ }^{5}$ polystyrenesulfonic acid doped with poly(3,4-ethylene dioxythiophene) (PEDOT-PSS), ${ }^{6-8}$ and PSS doped with polyaniline (Pani-PSS). ${ }^{7,8}$ Based on ultraviolet photoemission spectroscopic (UPS) study, Mäkinen et al. ${ }^{9}$ have proposed glycerol-modified PEDOT:PSS (hereafter, refer as the G-PEDOT layer)/hole-transporting material structure as one potential component in an OLED device. Indeed, promising device performance of small moleculebased OLEDs utilizing G-PEDOT in the anode structure has been reported. ${ }^{10,11}$ However, similar usage of G-PEDOT in PLEDs has not been reported. It is thus of interest to study the device performance of this modified PEDOT based PLEDs.

In this work, we fabricated polymer devices with the configuration of ITO/G-PEDOT/poly (9,9-dioctylfluorene) (F8)/CsF/Al to study the performance of the G-PEDOT buffer layer. In addition, we also carried out a corresponding UPS study on the interface between the G-PEDOT buffer layer and the F8 polymer to see how the G-PEDOT layer influences the hole-injection barrier.

The chemical structures of F8, PEDOT:PSS, and glycerol are shown in Fig. 1(a). G-PEDOT solutions with different glycerol concentrations were prepared by mixing glycerol $(0.6 \mathrm{~g}$ in $1 \mathrm{ml}$ of methanol) into $2-20 \mathrm{ml}$ of PEDOT:PSS, as mentioned in Ref. 9. Unmodified PEDOT: PSS or G-PEDOT were spin-coated on ultraviolet ozone treated prepatterned ITO substrates to form films of $60 \mathrm{~nm}$ thick. After baking the PEDOT:PSS or G-PEDOT films at

${ }^{\text {a)} E l e c t r o n i c ~ m a i l: ~ a p c s l e e @ c i t y u . e d u . h k ~}$ $150^{\circ} \mathrm{C}$ in a dry box for $1 \mathrm{~h}$, the emissive polymer, $\mathrm{F} 8$, was then spin-coated from a xylene solution $(1.5 \mathrm{wt} \%)$ to form a 70-nm-thick film. The samples were then loaded into a vacuum chamber for cathode deposition using a shadow mask. The thickness values of the deposited layers, as monitored by a calibrated quartz oscillating crystal, were 4 and $200 \mathrm{~nm}$ for $\mathrm{CsF}$ and $\mathrm{Al}$, respectively. Current densityvoltage-luminance $(J-V-B)$ characteristics and electroluminescent (EL) spectra of the fabricated devices were measured with a PR650 SpectraScan. There were four emissive pixels in the device and each of them had an emissive area of approximately $0.1 \mathrm{~cm}^{2}$.

For the UPS study, ITO substrates spin-coated with 10nm-thick film of completely dried PEDOT:PSS and G-PEDOT together with another two samples with an extra 5 -nm-thick F8 film on top ${ }^{12}$ were loaded into an ultrahighvacuum analysis chamber $\left(\sim 5 \times 10^{-10} \mathrm{mbar}\right)$ of a VG ESCALAB 220i-XL surface analysis system. Before the UPS analysis and the deposition of Cs, the film surface was degassed slightly at $80^{\circ} \mathrm{C}$ in situ. As the UPS spectra (not shown here) of the PEDOT:PSS and G-PEDOT anodes possess a decreasing density of states instead of a distinct edge near the Fermi level position, it was difficult to determine accurately the position of the Fermi edge in these highly conducting polymers. ${ }^{13,14}$ Thus, the work functions of the PEDOT:PSS and G-PEDOT were measured from the vacuum level onset and the spectrometer Fermi level position. It should be noted that in our UPS measurement we studied the $\mathrm{F} 8 / \mathrm{Cs}$ interface instead of the $\mathrm{F} 8 / \mathrm{CsF} / \mathrm{Al}$ interfaces, since (i) the role of $\mathrm{Al}$ on $\mathrm{CsF}$ is known to release $\mathrm{Cs}$ into the polymer, ${ }^{15}$ and (ii) the same report verified that deposition of $\mathrm{Al}$ onto a high photoelectron yield insulating layer of $\mathrm{CsF}$ induced charging which then smeared out some valence band features in the UPS. ${ }^{15}$

An energy level diagram summarizing the electronic structure of the interfaces in the ITO/PEDOT:PSS/F8/Cs and 
(a)

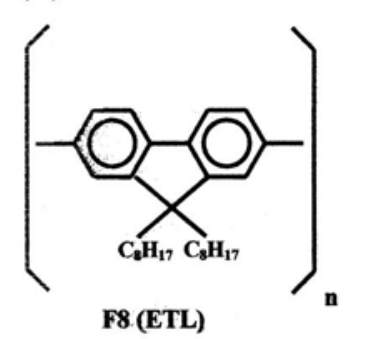

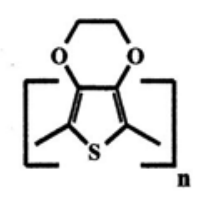

PEDOT:PSS (HTL:Dopant)

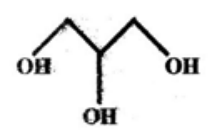

Glycerol (b)

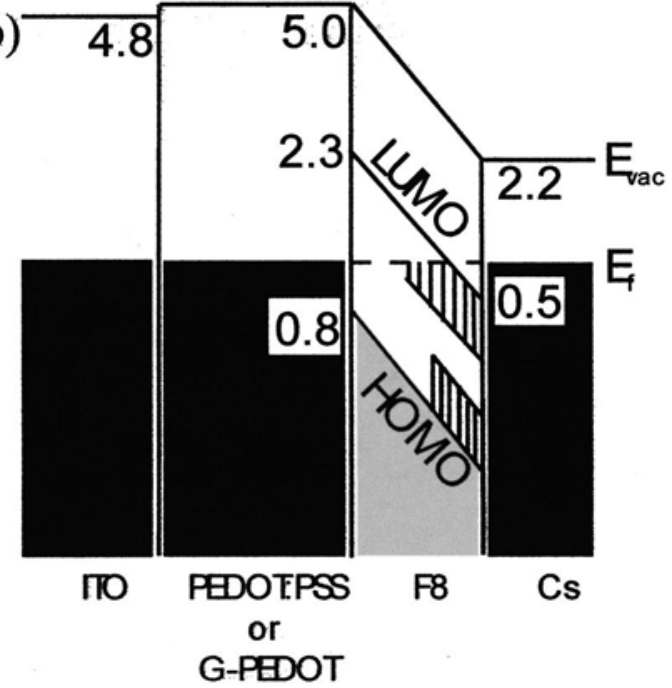

FIG. 1. (a) The chemical structure of F8, PEDOT:PSS, and glycerol. (b) An energy level diagram showing ITO/PEDOT:PSS (G-PEDOT)/F8/Cs interfaces. $E_{\mathrm{vac}}, E_{f}$, HOMO, and LUMO correspond to vacuum level, Fermi level, highest occupied molecular orbital and lowest unoccupied molecular orbital, respectively. The gap states known as bipolarons are also shown in the shaded regions.

ITO/G-PEDOT/F8/Cs structures is depicted in Fig. 1(b). The work functions of ITO, PEDOT:PSS, and Cs are 4.8, 5.0 , and $2.2 \mathrm{eV}$, respectively. The incorporation of glycerol into the PEDOT:PSS has no observable effect on the work function. Upon the Cs deposition, two gap states (known as bipolaron states) emerged in the UPS spectra of F8. The gap states are schematically shown in Fig. 1(b). The nature of these states and causes for their formations were described elsewhere. ${ }^{16,17}$ Another interesting finding is that the vacuum levels between PEDOT:PSS/F8, G-PEDOT/F8, and F8/Cs are always aligned and, hence, the interfacial dipoles $(\Delta s)$ obtained by $\phi_{\mathrm{PA}}-\phi_{\mathrm{F} 8}$ or $\phi_{\mathrm{F} 8}-\phi_{\mathrm{Cs}}$, where $\phi_{\mathrm{PA}}, \phi_{\mathrm{F} 8}$, and $\phi_{\mathrm{Cs}}$ represent the work function of the polymer anode, F8 and Cs cathode respectively, are zero. These results are consistent with the previous study. ${ }^{13}$ Accordingly, using a band gap value of $3.1 \mathrm{eV}^{18}$ and an ionization potential of $5.8 \mathrm{eV}$ for the pristine $\mathrm{F} 8$, the hole injection barriers at both the PEDOT:PSS/F8 and G-PEDOT/F8 interfaces are $0.8 \mathrm{eV}$. On the other hand, the electron injection barrier at the $\mathrm{F} 8 / \mathrm{Cs}$ interface is $-0.5 \mathrm{eV}$ (negative means effectively no barrier). The much higher hole-injection barrier suggests that the efficiency of these PLEDs is more affected by hole injection.

The F8-based devices exhibited two EL peaks at 440 and $470 \mathrm{~nm}$, as shown in the inset of Fig. 2, corresponding to blue emission. The introduction of the glycerol into PEDOT: PSS did not alter the positions of the emission peaks. The $J-V-B$ characteristics of the devices (1) ITO/PEDOT:PSS/ $\mathrm{F} 8 / \mathrm{CsF} / \mathrm{Al}$ (square) and (2) ITO/G-PEDOT/F8/CsF/Al (circle) are depicted in Fig. 2. Both devices exhibited a turn-on voltage (the operating voltage at $1 \mathrm{Cd} / \mathrm{m}^{2}$ ) of $3.1 \mathrm{~V}$. The turn-on voltage is basically determined by the injection barrier of the majority charge carrier, which is electron in the present case. ${ }^{19,20}$ As the two devices have the same polymer/ cathode interface, the same turn-on voltage is expected.

While the glycerol doping does not cause significant changes to the interfacial structure, it does modify the device characteristic considerably. It can be seen from the Fig. 2 that at a driving voltage of $6 \mathrm{~V}$, the current density in the glycerol-doped device $\left(76 \mathrm{~mA} / \mathrm{cm}^{2}\right)$ is double that in the undoped device $\left(38 \mathrm{~mA} / \mathrm{cm}^{2}\right)$. The brightness of the doped de- vice $\left(1300 \mathrm{Cd} / \mathrm{m}^{2}\right)$ is more than double that of the undoped device $\left(500 \mathrm{Cd} / \mathrm{m}^{2}\right)$. Since the hole-injection barriers are the same in the two devices, the enhancement of charge injection and luminescence may be either due to a smoother surface and hence a better electrical contact with the ITO or an increase of conductivity upon glycerol doping, as suggested by Mäkinen. ${ }^{9}$ The former presumption was disavowed. ${ }^{21}$ To verify the latter factor, we have measured the conductivities of the PEDOT:PSS and the G-PEDOT films. Additional devices with the configurations of ITO/PEDOT:PSS/F8/Au and ITO/G-PEDOT/F8/Au were also fabricated using gold as the cathode. The high work function of Au will result in inefficient electron injection. Thus, currents measured in the gold-cathode devices would effectively reflect the hole currents injected from the anodes.

It was found that the undoped PEDOT:PSS had a conductivity of $10 \mathrm{~S} / \mathrm{cm}$, which is consistent with that reported in another study. ${ }^{22}$ Upon glycerol incorporation, the conductivity was increased by six times to $\sim 60 \mathrm{~S} / \mathrm{cm}$. The inset of Fig. 2 also shows the $I-V$ characteristics of the gold-cathode devices. It can be seen that the resistance of the ITO/G$\mathrm{PEDOT} / \mathrm{F} 8 / \mathrm{Au}(2.7 \mathrm{M} \Omega$ ) is about five times lower than that of the ITO/PEDOT:PSS/F8/Au device. These results suggest that adding glycerol can substantially increase the conductivity of the PEDOT:PSS layer, and a corresponding increase in the hole current in the devices. It was also noted that at high applied voltages $(>15 \mathrm{~V})$ there was a bulging feature in the $I-V$ curves. It may arise from the leakage current due to one or a combination of the three factors: (i) electrons captured by defect sites, (ii) electrical charges directly tunneled from the highly conducting polymer to the ITO, and (iii) high electric field generated at the prepatterned ITO edge. Nevertheless, the leakage current at lower applied voltages as seen in the present plots is not significant.

Figure 3 shows the luminance and power efficiency of the two devices with the $\mathrm{CsF} / \mathrm{Al}$ cathode. At a current density of $20 \mathrm{~mA} / \mathrm{cm}^{2}$, the G-PEDOT/F8/CsF/Al device shows much higher power $(1.2 \mathrm{~lm} / \mathrm{W})$ and current $(1.8 \mathrm{Cd} / \mathrm{A})$ efficiencies than those $(0.8 \mathrm{~lm} / \mathrm{W}$ and $1.3 \mathrm{Cd} / \mathrm{A}$, respectively $)$ of 


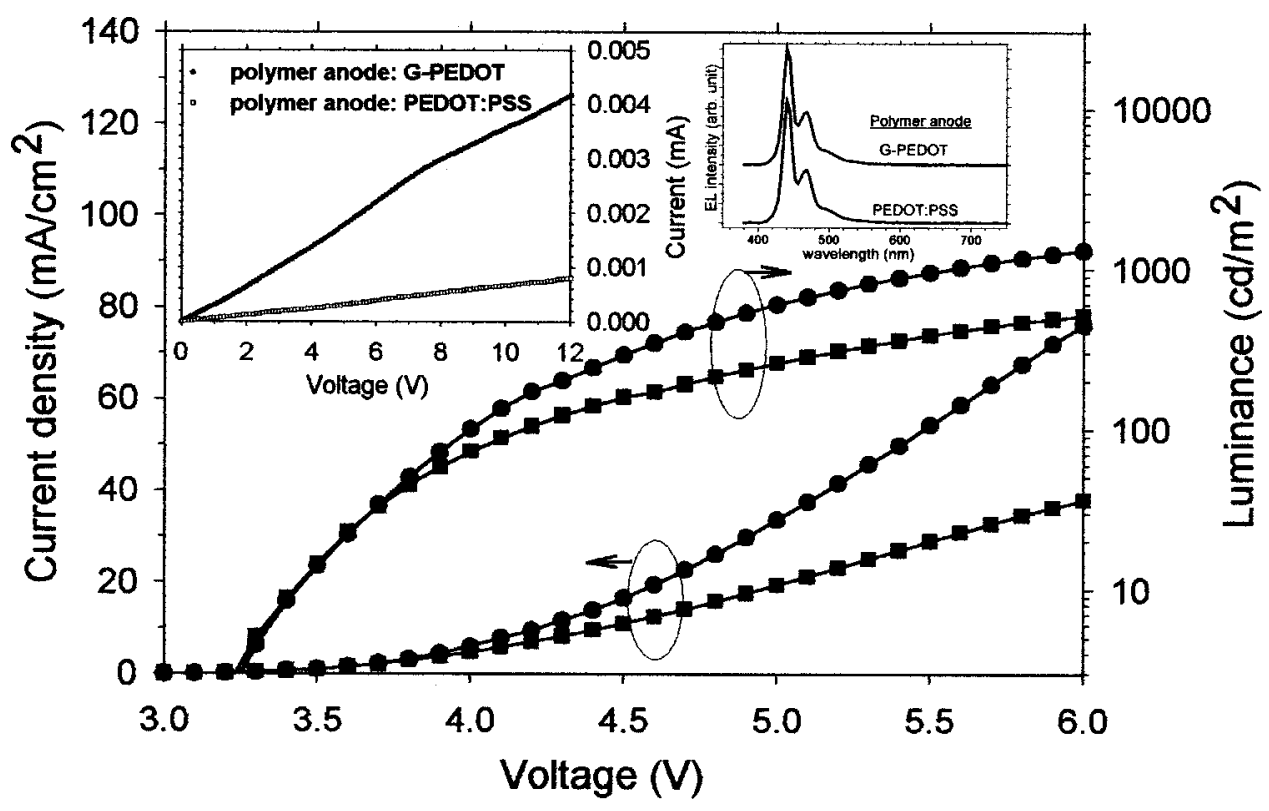

FIG. 2. $J-V-B$ characteristics of the devices ITO/PEDOT:PSS $(60 \mathrm{~nm}) / \mathrm{F} 8$ $(70 \mathrm{~nm}) / \mathrm{CsF} \quad(4 \mathrm{~nm}) / \mathrm{Al}(200 \mathrm{~nm})$ (square) and ITO/G-PEDOT (60 $\mathrm{nm}) / \mathrm{F} 8(70 \mathrm{~nm}) / \mathrm{CsF}(4 \mathrm{~nm}) / \mathrm{Al}(200$ nm) (circle). The inset shows (i) the corresponding normalized EL spectra, and (ii) $I-V$ characteristics of the hole-only devices ITO/PEDOT:PSS $(60 \mathrm{~nm}) / \mathrm{F} 8 \quad(70 \mathrm{~nm}) / \mathrm{Au} \quad(200 \mathrm{~nm})$ (open square) and ITO/G-PEDOT (60 $\mathrm{nm}) / \mathrm{F} 8(70 \mathrm{~nm}) / \mathrm{Au}(200 \mathrm{~nm})$ (filled circle).

the PEDOT:PSS/F8/CsF/Al device. As mentioned earlier, the device efficiency in the present study is basically limited by the hole injection. It is believed that the device configuration using G-PEDOT/F8/CsF/Al facilitates hole transport, which leads to better balance in the electron and hole currents and improved efficiency. The improvements on the F8-based PLEDs show that the application of glycerol-doped PEDOT: PSS as a conducting polymer anode is not only favorable to G-PEDOT/small molecule-based ${ }^{9}$ but also to G-PEDOT/ polymer-based light-emitting devices. It is reasonable that the glycerol-doped PEDOT:PSS might also be useful in other organic electrical, electronic, or optoelectronic devices.

In summary, we have fabricated a blue PLED with a power and current efficiency of $1.2 \mathrm{~lm} / \mathrm{W}$ and $1.8 \mathrm{Cd} / \mathrm{A}$, respectively, at a current density of $20 \mathrm{~mA} / \mathrm{cm}^{2}$ by using $\mathrm{F} 8$ sandwiched between the glycerol-modified PEDOT:PSS and CsF/Al. Comparing to the PLED using the undoped PEDOT: PSS, we found that the doped device doubled the current injection from 38 to $76 \mathrm{~mA} / \mathrm{cm}^{2}$ at a voltage of $6 \mathrm{~V}$. This enhancement was accompanied by a prominent increase of luminescence from 500 to $1300 \mathrm{Cd} / \mathrm{m}^{2}$ and current efficiency from 1.3 to $1.7 \mathrm{Cd} / \mathrm{A}$. UPS study revealed that the low work

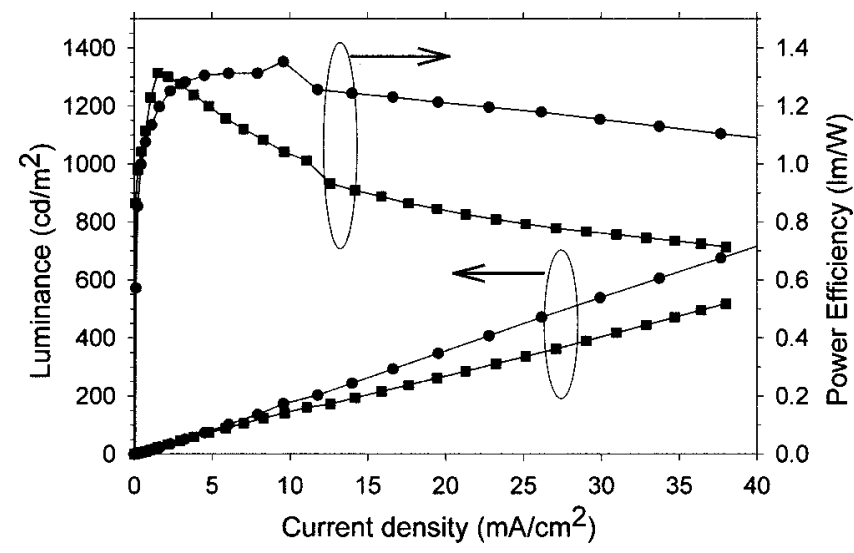

FIG. 3. Luminescence and power efficiency plotting against the current density, for the devices ITO/PEDOT:PSS $(60 \mathrm{~nm}) / \mathrm{F} 8(70 \mathrm{~nm}) / \mathrm{CsF}(4$ $\mathrm{nm}) / \mathrm{Al}(200 \mathrm{~nm})$ (square) and ITO/G-PEDOT $(60 \mathrm{~nm}) / \mathrm{F} 8(70 \mathrm{~nm}) / \mathrm{CsF}(4$ $\mathrm{nm}) / \mathrm{Al}(200 \mathrm{~nm})$ (circle). function Cs led to practically no electron injection barrier at the F8 interface and the better device performance arose from the increase in conductivity of the G-PEDOT layer.

This work was supported by the Research Grants Council of Hong Kong (Project CityU 1028/00E).

${ }^{1}$ T. M. Brown, R. H. Friend, I. S. Millard, D. J. Lacey, J. H. Burroughes, and F. Cacialli, Appl. Phys. Lett. 79, 174 (2001).

${ }^{2}$ R. B. Fletcher, D. G. Lidzey, D. D. C. Bradley, S. Walker, M. Inbasekaran, and E. P. Woo, Synth. Met. 111-112, 151 (2000).

${ }^{3}$ L. C. Palilis, D. G. Lidzey, M. Redecker, D. D. C. Bradley, M. Inbasekaran, E. P. Woo, and W. W. Wu, Synth. Met. 111-112, 159 (2000).

${ }^{4}$ F. Zhu, B. Low, K. Zhang, and S. Chua, Appl. Phys. Lett. 79, 1205 (2001).

${ }^{5}$ W.-L. Yu, J. Pei, Y. Cao, and W. Huang, J. Appl. Phys. 89, 2343 (2001).

${ }^{6}$ T. M. Brown, J. S. Kim, R. H. Friend, F. Cacialli, R. Daik, and W. J. Feast, Appl. Phys. Lett. 75, 1679 (1999).

${ }^{7}$ S. A. Carter, M. Angelopoulous, S. Karg, P. J. Brock, and J. C. Scott, Appl. Phys. Lett. 70, 2067 (1997).

${ }^{8}$ J. C. Scott, S. A. Carter, S. Karg, and M. Angelopoulous, Synth. Met. 85, 1197 (1997).

${ }^{9}$ A. J. Mäkinen, I. G. Hill, R. Shashidhar, N. Nikolov, and Z. H. Kafafi, Appl. Phys. Lett. 79, 557 (2001).

${ }^{10}$ W. H. Kim, A. J. Mäkinen, N. Nikolav, R. Shashidhar, H. Kim, and Z. H. Kafafi, Proc. SPIE 4464, 85 (2002).

${ }^{11}$ W. H. Kim, A. J. Mäkinen, N. Nikolav, R. Shashidhar, H. Kim, and Z. H. Kafafi, Appl. Phys. Lett. 80, 3844 (2002).

${ }^{12}$ Instead of using $1.5 \mathrm{wt} \%$ of $\mathrm{F} 8$ which was commonly used in device fabrications, $0.15 \mathrm{wt} \%$ of F8 was prepared for the UPS studies so as to eliminate the attainable charging.

${ }^{13}$ G. Greczynski, K. Kugler, and W. R. Salaneck, J. Appl. Phys. 88, 7187 (2000).

${ }^{14}$ G. Greczynski, Th. Kugler, M. Keil, W. Osikowicz, M. Fahlman, and W. R. Salaneck, J. Electron Spectrosc. Relat. Phenom. 121, 1 (2001).

${ }^{15}$ G. Greczynski, M. Fahlman, and W. R. Salaneck, J. Chem. Phys. 114, 8628 (2001)

${ }^{16}$ W. R. Salaneck, R. H. Friend, and J. L. Brédas, Synth. Met. 94, 173 (1998).

${ }^{17}$ M. K. Fung, S. L. Lai, S. N. Bao, C. S. Lee, J. J. O’Brien, M. Inbasekaran, W. W. Wu, and S. T. Lee, Synth. Met. 128, 97 (2002).

${ }^{18}$ L. S. Liao, M. K. Fung, C. S. Lee, S. T. Lee, M. Inbasekaran, E. P. Woo, and W. W. Wu, Appl. Phys. Lett. 76, 3582 (2000).

${ }^{19}$ I. D. Parker, J. Appl. Phys. 75, 1656 (1994).

${ }^{20}$ L. S. Liao, L. F. Cheng, M. K. Fung, C. S. Lee, S. T. Lee, M. Inbasekaran, E. P. Woo, and W. W. Wu, Chem. Phys. Lett. 325, 405 (2000).

${ }^{21}$ A. J. Mäkinen, 46th International Symposium on Optical Science and Technology, San Diego, California, 29 July-3 August 2001.

${ }^{22}$ A. N. Aleshin, S. R. Williams, and A. J. Heeger, Synth. Met. 94, 173 (1998). 